\title{
Saturation Intensity and Time Response of InGaAs-InGaP MQW Optical Modulators
}

\author{
M. E. Watson, J. L. A. Chilla, J. J. Rocca, J.-W. Kim, D. L. Lile, T. J. Vogt, and G. Y. Robinson, Senior Member, IEEE
}

\begin{abstract}
We report modulation saturation and time response measurements on InGaAs-InGaP MQW modulators. The measurements yield a saturation intensity of $(3.7 \pm 0.1) \mathrm{kW} / \mathrm{cm}^{2}$ for a $0-10 \mathrm{~V}$ swing and switching times between 10 and 90 ns, depending on the bias voltage and incident light intensity. The observed dependence indicates that field screening due to carrier build-up is the dominant physical mechanism determining both the speed and the saturation intensity. This conclusion is supported by results of theoretical calculations.
\end{abstract}

\section{INTRODUCTION}

NCREASING demands for high-speed optical switching devices has sparked considerable interest in the area of addressable optical modulators. Much success has come from work on multiple quantum well modulators utilizing the quantum confined Stark effect (QCSE). Material systems which have been studied for this purpose include GaAs-AlGaAs [1]-[3] InGaAs-AlGaAs [4], [5], InGaAs-GaAs [6]-[8], and InGaAs-GaAsP [9]. Recently, p-i-n modulators based on strained layers of InGaAs-InGaP grown on GaAs have also been demonstrated [10]. The InGaAs-InGaP modulators, which offer the advantage of a transparent GaAs substrate, were shown to require low bias voltages $(\sim 5 \mathrm{~V})$ for operation, and hence reduced power dissipation. Modulation of the absorption at the wavelength of the excitonic peak up to $25 \%$ has been obtained for a single transmission pass. However, two important device parameters for its utilization in applications: the saturation intensity and time response had not been studied.

In this paper, we report the results of a study of saturation intensity and time response for nonresonant InGaAs-InGaP MQW modulators and analyze the dominant physical mechanisms responsible for the observed device performance. The MQW modulators used in the experiments are $\mathrm{p}$-i-n diodes with the intrinsic region comprised of 24.5 periods of undoped, strain compensated $\mathrm{In}_{x} \mathrm{Ga}_{1-x} \mathrm{As}-\mathrm{In}_{y} \mathrm{Ga}_{1-y} \mathrm{P}$ quantum wells fabricated using gas source MBE [10]. The mole fractions used were $x=0.15$ and $y=0.5$. The top and bottom of the modulators have InGaP buffer layers $0.5 \mu \mathrm{m}$ thick. The quantum well thickness is $10 \mathrm{~nm}$ and the barrier thickness is $15 \mathrm{~nm}$. The barrier thickness was chosen to provide adequate tensile strain to compensate the compressive strain of the mismatched

Manuscript received April 29, 1994; revised August 10, 1994. This work was supported by the National Science Foundation, Center for Optoelectronic Computer Systems through NSF Grant ECD 9015128, by the Colorado Advanced Technology Institute Grant 1537757 and by the Air Force Office of Scientific Research (Contract F49620-93-1)

The authors are with the Center for Optoelectronic Computing Systems and Department of Electrical Engineering, Colorado State University, Fon Collins, CO 80523 USA.

IEEE Log Number 9407803. well layers, and thus avoid the formation of misfit dislocations. The devices were grown with $360 \mu \mathrm{m} \times 510 \mu \mathrm{m}$ active areas, $\mathrm{Au}-\mathrm{Ti}$ contacts, and $\mathrm{SiO}_{2}$ anti-reflection coatings. The samples used were from an array of modulators on the same region of a wafer which was configured for transmission measurements.

The next section discusses the saturation intensity measurements and Section III the results of the time response measurements. Finally, Section IV presents a simple model for the carrier sweep-out that describes well the observed intensity and voltage dependence of the transmission of the devices and gives values of the saturation intensity and time response of the modulation which are similar to those obtained experimentally.

\section{MEASUREMENTS OF THE SATURATION INTENSity}

The performance of modulators at high intensity is affected by several phenomena caused by the high density of photogenerated carriers including phase space filling [3], field screening due to charge build-up [11], [12], and thermal effects [13]. To determine the saturation intensity, we measured the transmission of the modulator at the excitonic wavelength as a function of incident light intensity. For this measurement we used a tunable CW Ti:Sapphire laser pumped by a CW argon ion laser as the light source. The light detection was done using $p-i-n$ silicon photodiodes and standard lock-in detection techniques. To minimize heating of the sample, the output of the laser was modulated using a specially constructed optical chopper to achieve pulses of $\sim 4 \mu$ s duration with a repetition rate of $\sim 3.5 \mathrm{kHz}$. The beam was focused on the sample utilizing a $5 \mathrm{~cm}$ focal length lens and the sample was positioned at the focal plane. The beam profile was measured to be approximately Gaussian and circular, with a $1 / e$ intensity radius of $10 \mu \mathrm{m}$ at the focus. The power density on the sample was adjusted by selecting the power of the laser using a variable neutral density filter.

Fig. 1 shows the transmission spectra of the InGaAs-InGaP modulators for applied voltages of 0 and $6 \mathrm{~V}$ of reverse bias for two different optical powers. For low intensity $\left(75 \mathrm{~W} / \mathrm{cm}^{2}\right)$ and no bias applied to the device, the excitonic resonance is clearly visible as an absorption peak at $960 \mathrm{~nm}$. Applying 6 $\mathrm{V}$ to the device, the excitonic peak shifts and broadens due to the quantum confined Stark effect, resulting in a net change in transmission at the excitonic wavelength that allows the use of this device as a modulator at that wavelength. With a $10 \mathrm{~V}$ swing in applied bias, the contrast ratio is 1.25 which could be increased by configuring the device for reflection operation [10]. Fig. 1(b) shows the effect of high intensity $\left(2.5 \mathrm{~kW} / \mathrm{cm}^{2}\right)$ on the transmission spectrum of the device. The $0 \mathrm{~V}$ data still 


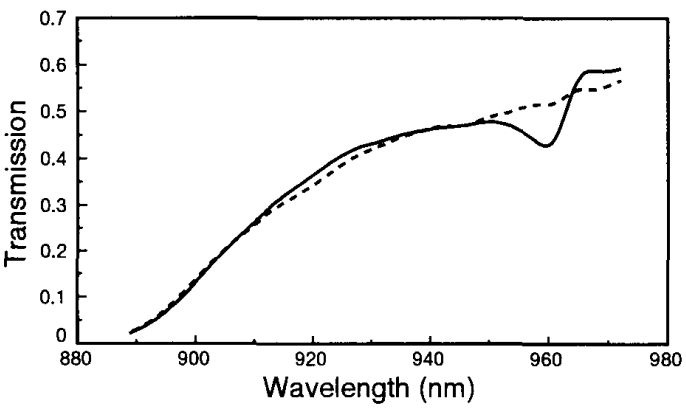

(a)

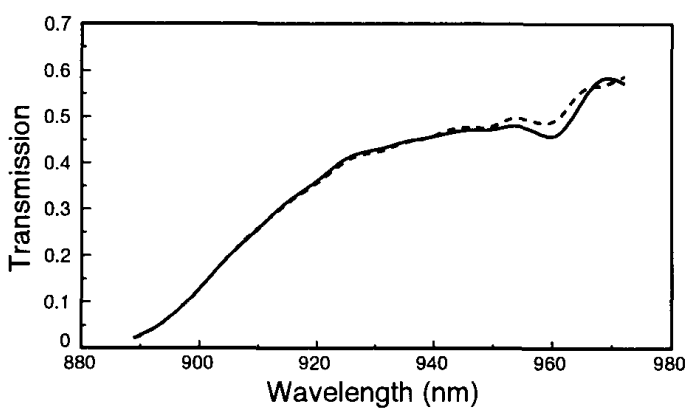

(b)

Fig. 1. Transmission spectra of the modulator measured at two different intensities. $75 \mathrm{~W} / \mathrm{cm}^{2}$ (a) and $2.5 \mathrm{~kW} / \mathrm{cm}^{2}$ (b), and applied voltages, 0 (solid line) and $6 \mathrm{~V}$ (dashes).

shows the excitonic peak, although not as distinct as in the low intensity case. The change in transmission with applied voltage is also smaller at high intensity, thus reducing the contrast ratio of the device.

From a technological point of view, and regardless of the physical origin of this saturation, it is necessary to define a parameter that characterizes the dependence of the contrast ratio $C R$ with incident intensity $I$. As done in [14], we will define the modulation saturation intensity $I_{\text {sat }}$ according to the following expression

$$
\mathrm{CR}(I)=1+\frac{\mathrm{CR}(0)-1}{1+I / I_{\mathrm{sat}}} .
$$

The measured dependence of the contrast ratio with incident power density was fitted with expression (1) for voltage swings ranging from $(0-3 \mathrm{~V})$ to $(0-10 \mathrm{~V})$. Fig. 2 shows the change in contrast ratio corresponding to a $0-10 \mathrm{~V}$ bias swing for four different devices for input intensities between 0.1 and 60 $\mathrm{kW} / \mathrm{cm}^{2}$. As can be observed in the figure, the dependence of the contrast ratio as a function of the light intensity is well described by expression (1) (solid line) for all four devices. The fitted value of saturation intensity for a $0-10 \mathrm{~V}$ swing is $(3.7 \pm 0.1) \mathrm{kW} / \mathrm{cm}^{2}$.

Fig. 3 shows the resulting dependence of modulation and saturation intensity with voltage. At low power, the modulation saturates at about $6 \mathrm{~V}$ (Fig. 3(a)). This is consistent with the fact that the excitonic absorption is washed out for voltages greater than $\sim 6 \mathrm{~V}[10]$. For higher power, the modulation remains linear with voltage within the $0-10 \mathrm{~V}$ range studied, but its maximum decreases due to saturation. The saturation

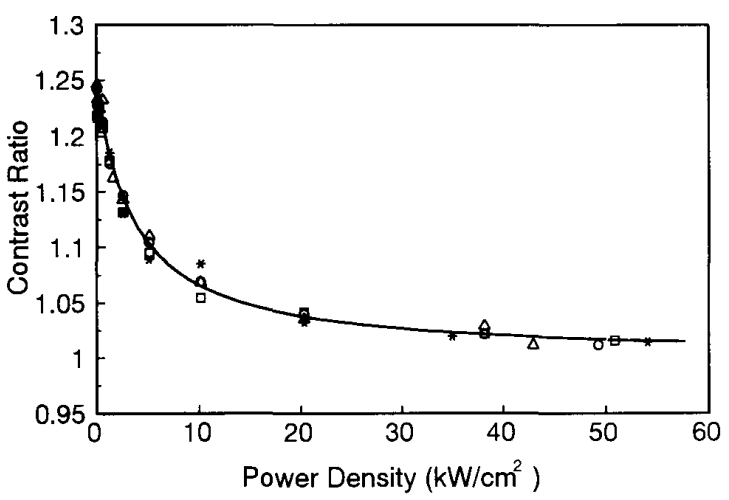

Fig. 2. Contrast ratio versus incident intensity for a $10 \mathrm{~V}$ change in applied voltage. The symbols represent different devices in the same wafer, the solid curve is a least squares fitting.

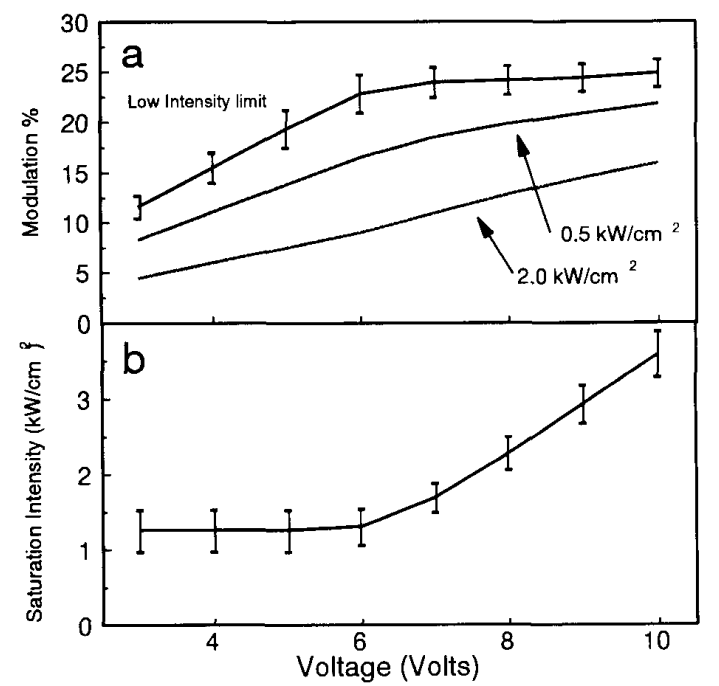

Fig. 3. Dependence of modulation (a) and saturation intensity (b) as a function of operating voltage.

intensity as a function of applied voltage is shown in Fig. 3(b). The saturation intensity is relatively constant with a value of $(1.3 \pm 0.1) \mathrm{kW} / \mathrm{cm}^{2}$ up to $\sim 6 \mathrm{~V}$ where the saturation begins to increase linearly with voltage to reach $(3.7 \pm 0.1) \mathrm{kW} / \mathrm{cm}^{2}$ at $10 \mathrm{~V}$. This saturation intensity value is relatively small when compared with that of similar devices constructed utilizing other material systems [3], [14], [15].

A physical mechanism affecting the performance of modulators at high light intensities is the saturation of the excitonic absorption [3]. Our measurements indicate that in these devices, this is not the dominant physical mechanism responsible for the observed saturation of the modulation. This can be concluded from Fig. 4. Fig. 4(a) shows the differential transmission spectra for a $0-6 \mathrm{~V}$ swing both at low and high intensity. The operating wavelength is indicated by a vertical dotted line in the figure. It is evident that degradation of the modulation occurs at high intensity. Fig. 4(b) shows the corresponding transmission spectra at 0 and $6 \mathrm{~V}$ for low and high intensity. The transmission at the operation wavelength 


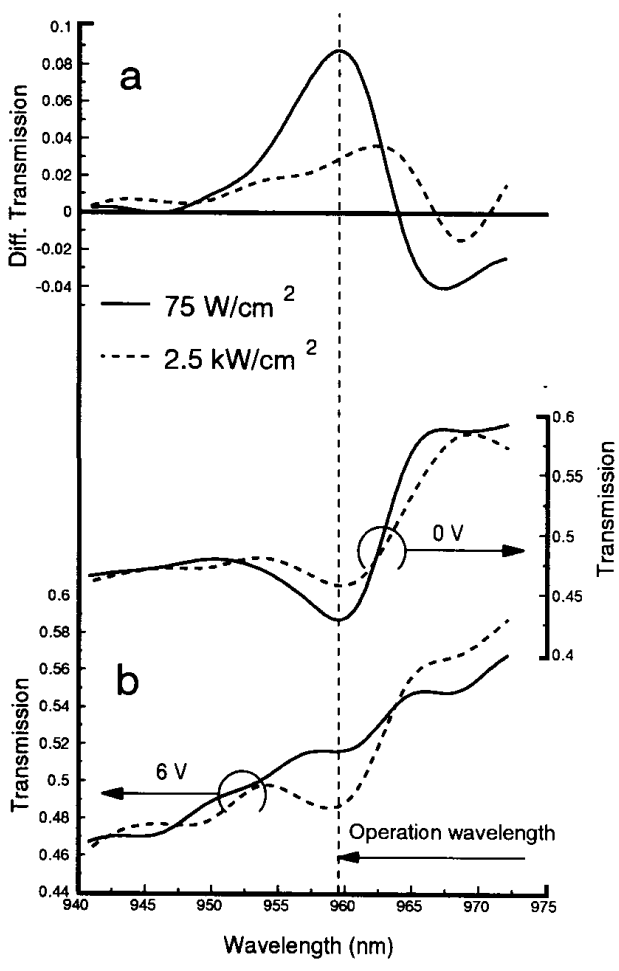

Fig. 4. (a) Differential transmission spectra at low and high intensities for a $0-6 \mathrm{~V}$ voltage swing. (b) Transmission spectra at 0 and $6 \mathrm{~V}$ for the same intensities as in (a).

drops noticeably at high intensity for the $6 \mathrm{~V}$ data, contrary to what would be produced by saturation of the excitonic absorption. We note that most of the saturation observed in the modulation is due to the change in transmission at $6 \mathrm{~V}$, suggesting that field screening due to charge build-up [11], [12] and/or thermal effects caused by ohmic heating associated with the photocurrent [13] are responsible for the observed saturation of the modulation. The role of these mechanisms will be discussed in the following sections.

As discussed in [3], there is a close relationship between the sweep out time of the carriers and the value of the saturation intensity. For that reason, time response measurements on the modulators not only have a value of their own in characterizing device performance, but also provide further evidence that allows the attribution of the observed saturation to field screening effects. The results of time response measurements are discussed in the following section.

\section{Time Response Measurements}

The time response of the device was characterized by measuring the change in transmission at the excitonic wavelength produced by fast ( $<4 \mathrm{~ns}$ risetime) electrical bias pulses applied to the device. Simultaneous measurement of the current shows that the capacitance of the device $(\sim 30-50 \mathrm{pF})$ is not a limitation for its speed in this range. The transmitted light was detected with a silicon photodetector having a response time of approximately $1 \mathrm{~ns}$. The signal was amplified by a fast ( 3 ns risetime) amplifier and was measured with a $250 \mathrm{MHz}$

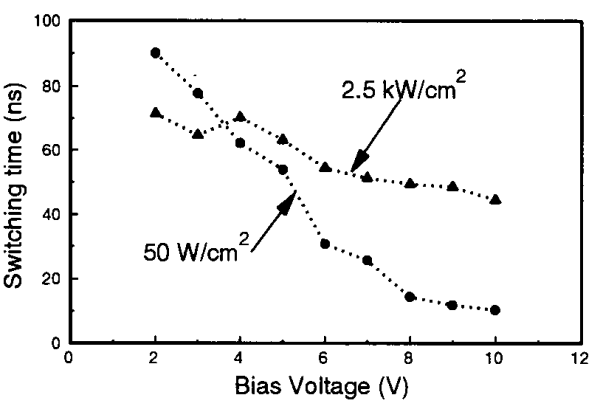

Fig. 5. Switching time $(10 \%-90 \%)$ versus operating voltage measured for $50 \mathrm{~W} / \mathrm{cm}^{2}$ and $2.5 \mathrm{~kW} / \mathrm{cm}^{2}$. The lines are only intended to guide the eye.

oscilloscope. The overall resolution of the system thus allows us to measure changes in transmission occurring in times of approximately $4 \mathrm{~ns}$.

We measured the switching of the transmission for different voltages and incident light intensities. The results are summarized in Fig. 5, where we plot the switching times (for $10 \%$ to $90 \%$ change in transmission) for applied voltages from 2 to 10 $\mathrm{V}$, at low and high incident intensities of $50 \mathrm{~W} / \mathrm{cm}^{2}$ and 2.5 $\mathrm{kW} / \mathrm{cm}^{2}$, respectively. For low intensity there is a significant reduction in the switching time from $90 \mathrm{~ns}$ at $2 \mathrm{~V}$ to $10 \mathrm{~ns}$ at $10 \mathrm{~V}$ as applied bias increases. In contrast, for high intensity the switching time remains relatively constant for all applied voltages. As discussed below, the observed dependence with voltage and intensity is consistent with the presence of field screening and rules out heating as the dominant mechanism. Let us note here that the measured switching times are slower than other devices previously reported in the literature [1], [11], which is consistent with a lower saturation intensity [3].

Heating effects were studied for $\mathrm{AlGaAs} / \mathrm{GaAs}$ p-i-n modulators [13]. As thermal properties are similar for all III-V materials, we would expect comparable results for a similar structure in the InGaP/InGaAs material system. As shown in [13], when heating is the dominant mechanism the time response of the transmission has two components, one fast corresponding to the onset of the applied field, and one slow corresponding to the change in transmission due to heating. As heat is produced mainly by ohmic dissipation caused by the photocurrent [13], the power to the device increases with applied voltage, making the slow component of the signal more noticeable as voltage increases. The decay time of the slow component was determined [13] to be close to $50 \mathrm{~ns}$ and should be independent of the applied voltage.

Although the measured switching times for our devices are in that range, our experiments do not show any of the features that can be attributed to heating, as can be observed in Fig. 6, which shows the temporal evolution of the transmission of the device for different conditions of incident intensity and bias voltage. All curves have been normalized to the steady state modulation for ease of comparison. Curves $a$ and $b$ were measured with $50 \mathrm{~W} / \mathrm{cm}^{2}$ of incident intensity, while curves c and $\mathrm{d}$ correspond to $2.5 \mathrm{~kW} / \mathrm{cm}^{2}$. The applied voltage changes from 0 to $10 \mathrm{~V}(\mathrm{a}, \mathrm{c})$ and 0 to $4 \mathrm{~V}(\mathrm{~b}, \mathrm{~d})$. The two component rise of the transmission is not observed in any of the curves, while the change in switching speed is evident. 


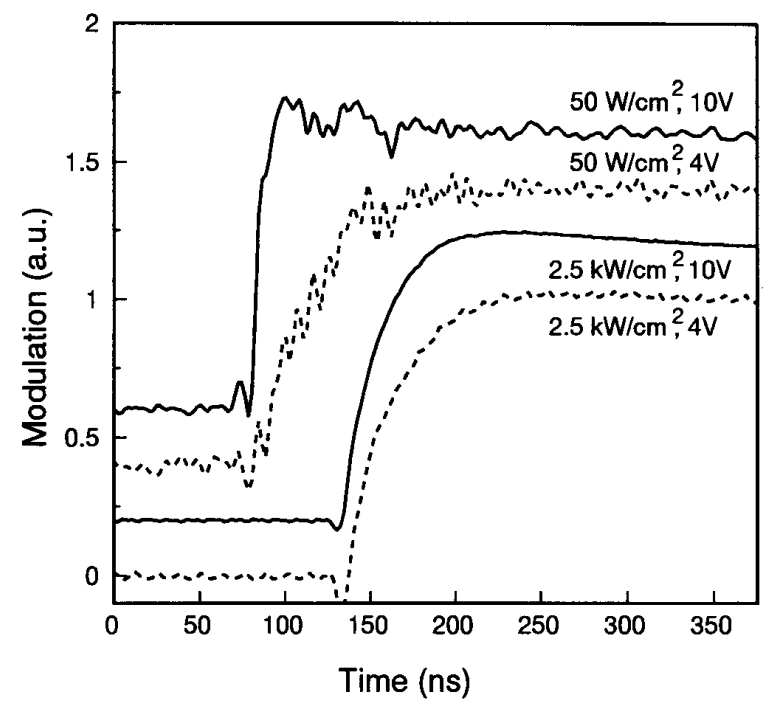

Fig. 6. Measured temporal evolution of the transmission of the device normalized to the steady state modulation. The applied voltage changes from 0 to the voltage in the figure with a risetime of $4 \mathrm{~ns}$, the intensity of the laser is also indicated in the figure.

Fig. 4 provides further evidence against the hypothesis of thermal effects because heat absorbed in the sample would produce a localized increase in lattice temperature which would shift the band gap and accompanying excitonic resonance towards lower energy. This shift would move the excitonic absorption out of the operation wavelength of the device, thus increasing the transmission, contrary to the behavior of the transmission shown in Fig. 4.

Field screening effects [11], [12] occur due to charge buildup of one of the photogenerated carriers: either electrons or holes. Carriers may escape from the quantum wells through thermionic emission or tunneling through the barrier. As it is likely that electrons escape much more rapidly than holes due to the difference in effective masses, accumulation of holes results in charge build-up. Tunneling escape probability is negligible because the barriers are $15 \mathrm{~nm}$ thick [3], leaving thermionic emission as the only escape process. The thermionic emission rate for holes [16] is given by

$$
\frac{1}{\tau}=\left(\frac{k_{B} T}{2 \pi m_{h w} L_{w}^{2}}\right)^{1 / 2} \exp \left(-\frac{H(F)}{k_{B} T}\right) .
$$

In this expression $T$ is the lattice temperature, $m_{h w}$ is the effective mass for holes in the well, $k_{B}$ is Boltzmann constant and $L_{w}$ is the well width. The barrier height $H$ depends on the field $F$ through

$$
H(F)=Q \Delta E_{g}-E_{h}-|e| F L_{w} / 2
$$

where $Q$ is the fraction of discontinuity in the valence band, $\Delta E_{g}$ is the difference in band gaps between the well and barrier material, $e$ is the electron charge and $E_{h}$ is the energy of the $n=1$ level for holes. Although measurements in $\mathrm{AlGaAs} / \mathrm{GaAs}$ [12] showed that the escape time is not accurately predicted by expression (2), it gives the correct qualitative behavior and is a good approximation for a simple model. Calculations assuming $60 \%$ [17] of the $\sim 0.67 \mathrm{eV}$ of bandgap difference [18] occurs in the valence band offset reveal that the hole thermionic lifetime varies between a few ns and $400 \mathrm{~ns}$ depending on the applied field. A similar time scale is to be expected for changes in the transmission. From (2) we can see that reduction of barrier height (through increased applied bias) results in faster escape times, in agreement with the low intensity data in Figs. 5 and 6. Also, for high intensity, the accumulated carriers would be expected to screen the field so that no change in speed would result, again in agreement with the observed behavior of the InGaAs-InGaP modulators. Similar effects have been observed in InGaAs-InP MQW modulators [11], on a much faster time scale, which agrees with estimates from (2).

We performed simulations using a simple model of the modulator that support the above qualitative reasoning that field screening is the dominant saturation mechanism in these devices.

\section{MODEL}

The model we used to simulate charge build-up is similar to that presented by Wood et al. [11]. We consider the reverse bias $\mathrm{p}-\mathrm{i}-\mathrm{n}$ structure as a capacitor with the quantum wells located between the plates. The differential equations governing the areal densities of electrons $n$ and holes $p$ in a quantum well illuminated with an intensity $I$ and subjected to a field $F$ are

$$
\begin{aligned}
& \frac{\partial n}{\partial t}=\alpha(F) I-B n p-n / \tau_{n} \\
& \frac{\partial p}{\partial t}=\alpha(F) I-B n p-p / \tau_{p} .
\end{aligned}
$$

Here $\alpha(F)$ is the field dependent absorption of one well, $B$ is the recombination constant and $\tau_{n}\left(\tau_{p}\right)$ is the electron (hole) escape time. The fact that the escape times of electrons and holes are different leads to accumulation of one kind of carrier in the well with the corresponding loss of charge neutrality. We will simulate this effect by the introduction of a layer of charge in the central plane of the quantum well with a charge density given by

$$
\sigma=e(p-n) .
$$

For high incident light intensity, the net positive charge can significantly distort the electric field in the depletion region. When the positive charge is large enough, the potential at one particular well may be reduced until it approaches the Fermi level of the $n$-type material. Under these conditions, electrons can diffuse from the bulk into that well, effectively reducing the width of the depletion region. In each step of the simulation, we determine the field using Gauss's law and the depletion width $d$ from the boundary condition:

$$
V_{b i}+V_{e}=\int_{0}^{d} F(x) d x
$$

where $V_{b i}$ and $V_{e}$ are respectively the built-in and external voltages. The background doping level in the intrinsic material of wells and barriers is assumed negligible for simplicity of the model, which is completed with charge layers at both ends of the depletion region. We number the wells starting from the 
TABLE I

Parameters Used in the Simulation

\begin{tabular}{|c|c|}
\hline Temperature & $T=300 \mathrm{~K}$ \\
\hline Bandgap difference & $\Delta E=673 \mathrm{meV}$ \\
\hline Fraction of discontinuity in valence band' & $Q=0.6$ \\
\hline Effective mass electrons in the well ${ }^{a}, f$ & $m_{\mathrm{all}}=0.0604 \times m_{0}$ \\
\hline Effective mass electrons in the barrier ${ }^{\text {a } f}$ & $m_{a b}=0.119 \times m_{0}$ \\
\hline Effective mass holes in the well ${ }^{\text {a.l }}$ & $m_{m, 1}=0.471 \times m_{0}$ \\
\hline Effective mass holes in the barrieriti & $m_{h b}=0.62 \times m_{0}$ \\
\hline Recombination constant & $B=10^{-4} \mathrm{~cm}^{2} / \mathrm{s}$ \\
\hline Diffusion time constant ${ }^{\mathrm{d} .,}$ & $T_{r l}=400 \mathrm{~ns}$ \\
\hline Well width & $L_{u}=10 \mathrm{~nm}$ \\
\hline Barrier width & $L_{b}=15 \mathrm{~nm}$ \\
\hline Energy of $n=1$ level for electrons & $E_{\mathrm{f}}=30.8 \mathrm{meV}$ \\
\hline Energy of $n=1$ level for holes & $E_{l_{l}}=26.3 \mathrm{meV}$ \\
\hline Dielectric constant & $f=12$ \\
\hline Area of the device & $A=360 \times 510 \mu \mathrm{m}^{2}$ \\
\hline Number of wells & $n=24$ \\
\hline Built in voltage & $T_{i j i}=1 \mathrm{~V}$ \\
\hline Focal spot radius & $r=10 \mu \mathrm{m}$ \\
\hline
\end{tabular}

Notes:

a) From [18]

b) From calculations following the procedure in [17].

c) Results in lifetime around $1 \mathrm{~ns}$.

d) Computed from a diffusion constant of $10 \mathrm{~cm}^{2} / \mathrm{s}$.

e) Order of magnitude from GaAs-AlGaAs [19] f) $m_{0}=$ electron mass.

one closest to the $p$ region of the diode and assume the light is traveling from $p$ to $n$.

The numerical values of the parameters used in the simulation are listed in Table I. The field dependent absorption is obtained from low intensity transmission measurements under conditions so as to make charge build-up effects negligible. Besides thermionic emission time, computed using expression (1), the escape time for both electrons and holes also includes tunneling, and diffusion of the carriers in the plane of the wells out of the illuminated area. Although it is negligible for our structure, tunneling was included in order to be able to evaluate different device designs and was computed using formulas given in [3]. The ambipolar diffusion time for a diffusion coefficient of $10 \mathrm{~cm}^{2} / \mathrm{s}$ [19] is comparable to thermionic emission when the field is low but, because of its ambipolar nature, it does not contribute to the charge build-up.

We first performed steady state calculations, in which we computed the mutually dependent field and charge distributions in a self consistent manner. Fig. 7 shows the steady state carrier density (a) and the field distribution (b) for the different wells in the structure. These results were obtained for intensities of $16 \mathrm{~W} / \mathrm{cm}^{2}$ and $2.5 \mathrm{~kW} / \mathrm{cm}^{2}$ while the external voltage was kept constant at $5 \mathrm{~V}$. In Fig. 7(a) the circles (triangles) represent the hole (electron) population while the intensity is denoted by the use of empty symbols for high intensity and solid symbols for low intensity. In Fig. 7(b) the result for low intensity is shown by the solid line and that for the high intensity by the dotted line.

In the depletion region, where the field is not zero (Fig. 7(b)), the electrons are efficiently swept out of the wells, and there is accumulation of holes (Fig. 7(a)) due to their

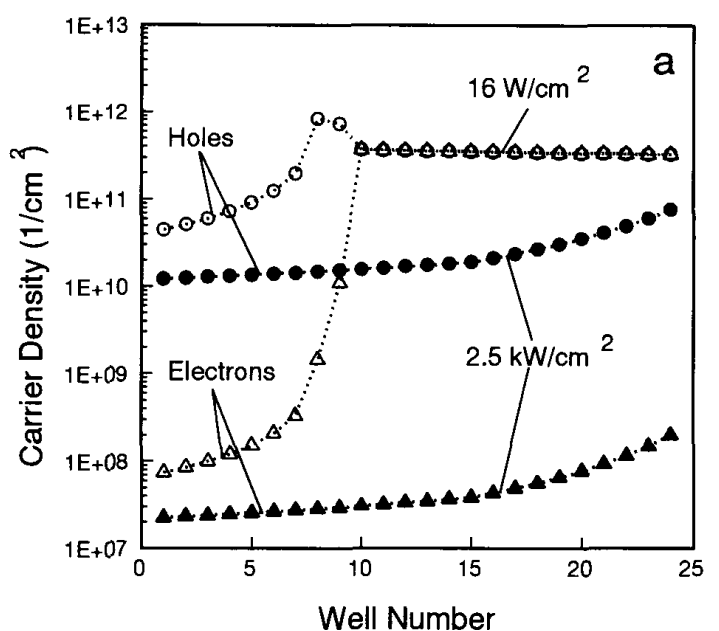

(a)

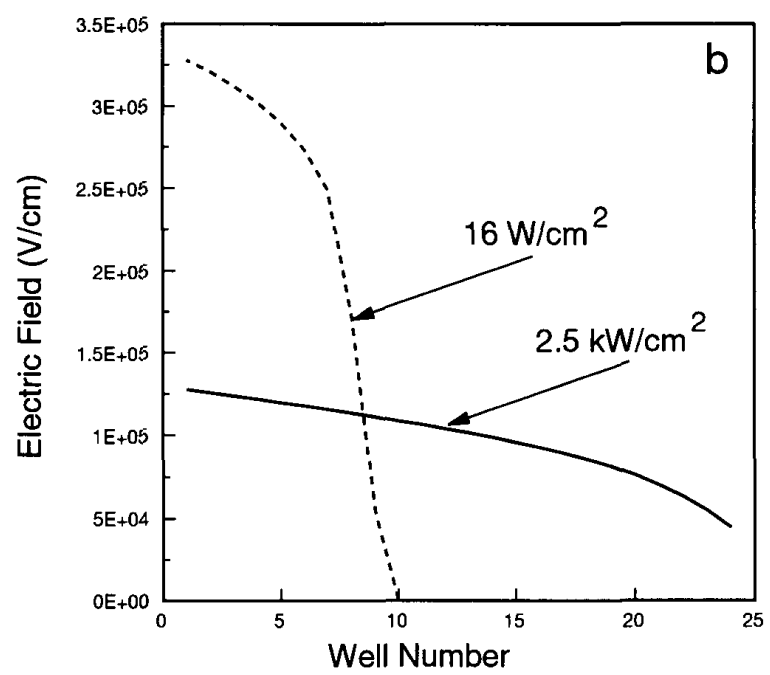

(b)

Fig. 7. (a) Computed steady state distribution of electron (triangles) and holes (circles) areal densities with an applied voltage of $5 \mathrm{~V}$ for intensities of $16 \mathrm{~W} / \mathrm{cm}^{2}$ (solid symbols) and $2.5 \mathrm{~kW} / \mathrm{cm}^{2}$ (empty symbols). The $p$ region is located to the left of the wells. The light travels from left to right. (b) Computed steady state electric field for the same conditions as in (a). Low intensity data is plotted with solid line, and high intensity data with dashed line.

longer escape time. For $5 \mathrm{~V}$ of external bias, and in absence of illumination, all the wells would be subjected to an electric field around $10^{5} \mathrm{~V} / \mathrm{cm}$. Due to the presence of net charge in the wells, the field is not the same for all the wells and those where the field is lower have higher absorption and longer escape times, leading to higher densities to the right end ( $n$ side) of the depletion region in Fig 7. The accumulation of carriers is not very important when the intensity is low, and most of the wells experience a value of the field close to that in the absence of light. Notable changes occur in the charge distribution when the intensity is increased. As the carrier density increases, the charge accumulates and the width of the depletion region varies accordingly. Once a well is out of the depletion region, the field goes to zero with a corresponding 
change in transmission and with equal populations of electrons and holes. As shown in Fig. 7(b), at high intensity only few of the wells experience the field, resulting in a reduction of the modulation with respect to the low intensity case. These qualitative results of the model show the same behavior that was observed in the experiment (Fig. 4). In spite of uncertainties in certain material parameters and its simplicity, the model also predicts the modulation with less than $20 \%$ error respect to the experimental values for intensities up to the measured $I_{\text {sat }}\left(\sim 4 \mathrm{~kW} / \mathrm{cm}^{2}\right)$. At higher intensities, however, the discrepancies are larger and it does not account for all the reduction of modulation that is observed. This difference might be due to effects not included in our model that are nevertheless suggested by the shape of the spectra (Figs. 1 and 4), such as slight saturation of the excitonic absorption or unintentional doping of the material in the intrinsic region. Another possible source of error is inaccuracy associated with the use of (2) to estimate the thermionic escape times.

The simulation of the time response of the modulators starts from the steady state situation for $V_{e}=0 \mathrm{~V}$, and follows the changes produced by an increase of applied voltage with a risetime of $4 \mathrm{~ns}$. In each step of the simulation, the following tasks are performed.

1) An estimate of the external charge flow needed to establish the new voltage across the device is computed. As the flow of carriers external to the MQW structure is much faster than the time scale of the escape from the wells, we assume that the external carriers flow so as to accommodate the internal changes in accordance to the boundary condition (7).

2) The rate of change of the carrier population in all the wells is computed by means of expression (4) and (5) and is used to calculate the new carrier densities after a short time interval $d t$. The total transmission coefficient of the structure is also computed.

3) The external charge is recomputed to meet the boundary condition (7) taking into account the new internal charge distribution. This is usually a small correction of the values calculated in step 1 . Also in this step the width of the depletion region is modified when the new internal charge distribution makes it necessary.

Typical results of the simulation are plotted in Fig. 8, which shows the simulated temporal evolution of the transmission of the device for different conditions of incident intensity and bias voltage. All curves have been normalized to the steady state modulation for ease of comparison. Curves a and $b$ correspond to $50 \mathrm{~W} / \mathrm{cm}^{2}$ of incident intensity, while curves $\mathrm{c}$ and $\mathrm{d}$ were computed using $2.5 \mathrm{~kW} / \mathrm{cm}^{2}$. The applied voltage changes from 0 to $10 \mathrm{~V}(\mathrm{a}, \mathrm{c})$ and 0 to $4 \mathrm{~V}(\mathrm{~b}, \mathrm{~d})$ with a risetime of $4 \mathrm{~ns}$. As can be seen by comparing these results with the corresponding experimental curves shown in Fig. 6 , the model reproduces qualitatively the temporal changes in transmissivity observed in the experiment. At low intensity (a and b), an increase in bias voltage is accompanied by a decrease in switching time, in agreement with the data in Figs. 5 and 6, while at high intensity (c and d) that change is much less noticeable, also in agreement with the experiment.

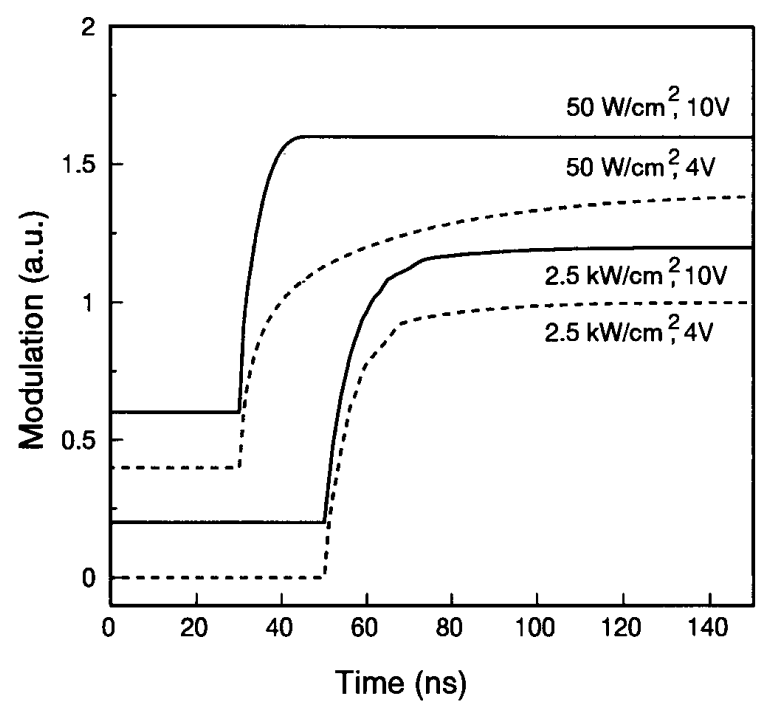

Fig. 8. Simulated temporal evolution of the transmission of the device normalized to the steady state modulation. The conditions of the simulation are the same as those of the experiment in Fig. 6.

The switching time for $10 \mathrm{~V}$ bias at high intensity is slower than that of $10 \mathrm{~V}$ at low intensity. The origin of this effect is the change in depletion width with voltage. As can be seen from the steady state data in Fig. 7(a), most of the carriers are accumulated near the end of the depletion region. A sudden increase of voltage moves the boundary of the depletion region closer to the $n$ region, leaving several wells in a situation out of equilibrium with low field and consequently $((1))$ long time to reach the new steady state situation.

While the simulation gives numerical values for the risetime which do not match exactly those obtained experimentally, this difference is again to be expected given the simplicity of the model and the fact that some of the parameters used are only estimates. Nevertheless, the results of the model give solid support to the qualitative reasoning presented in the previous section leading to the conclusion that field screening due to carrier build-up is the most important effect determining the observed saturation of the modulation in the InGaAs-InGaP modulators investigated. We also ran our simulation for a different structure, with barriers of $7.5 \mathrm{~nm}$ thickness. This change makes tunneling the dominant escape mechanism, and produces approximately a tenfold increase in saturation intensity and switching speed limited by the risetime of the electrical pulse. Although the simplicity of the model does not allow a detailed prediction, it shows the possibility of increase both the saturation intensity and speed of the device by means of changes in the structure.

\section{CONCLUSION}

We performed a detailed study of the performance of InGaAs-InGaP multiple quantum well modulators under different conditions of incident intensity and operating voltage. We found that the saturation intensity is relatively constant with a value of $(1.3 \pm 0.1) \mathrm{kW} / \mathrm{cm}^{2}$ up to $\sim 6 \mathrm{~V}$ bias where the saturation begins to increase linearly with 
voltage to reach $(3.7 \pm 0.1) \mathrm{kW} / \mathrm{cm}^{2}$ at $10 \mathrm{~V}$ bias. Spectra at different powers and applied voltages show that the decrease of modulation with power is not due to saturation of the excitonic absorption. We performed measurements on the time response of the devices, which not only characterizes the modulation speed of the device, but also confirm that the most important cause of the saturation is field screening due to charge build-up. The obtained switching times ranging between 10 and $90 \mathrm{~ns}$ are not limited by the capacitance of the device, but by the escape time of the photogenerated carriers from the wells. In the devices studied the escape time was dominated by thermionic emission, which is slower than in other material systems due to the larger difference in band gap between the well and barrier materials. In order to improve the speed of InGaAs-InGaP devices while keeping the advantage of a transparent substrate, the structure must be carefully engineered to accelerate the escape of the carriers from the wells, for example by using thinner barriers that would allow tunneling. We performed simulations using a simple model of the modulator which includes field screening effects that confirm field screening to be the dominant physical mechanism governing the saturation and time response of these devices.

\section{REFERENCES}

[1] T. H. Wood, C. A. Burrus, D. A. B. Miller, D. S. Chemla, T. C. Damen, A. C. Gossard, and W. Wiegmann, "High-speed optical modulation with GaAs/GaAlAs quantum wells in a p-i-n diode structure," Appl. Phys. Lett., vol. 44, pp. 16-18, 1984.

[2] T. K. Woodward, L. M. F. Chirovsky, A. L. Lentine, L. A. D'Asaro, E J Laskowski, M. Focht, G. Guth, S. S. Pei, F. Ren, G. J. Przybylek, L. E Smith, R. E. Leibenguth, M. T. Asom, R. F. Kopf, J. M. Kuo, and M. D. Feuer, "Operation of a fully integrated GaAs-AlxGal-xAs FET-SEED: A basic optically addressed integrated circuit," IEEE Photon Technol. Lett., vol. 4, pp. 614-617, 1992.

[3] A. M. Fox, D. A. B. Miller, G. Livescu, J. E. Cunningham, and W. Y. Jan, "Quantum well carrier sweep out: Relation to electroabsorption and exciton saturation," IEEE J. Quantum Electron., vol. 27, pp. 2281-2294, 1991.

[4] B. Pezeshki, S. M. Lord, and J. S. Harris, Jr. "Electroabsorptive modulators in InGaAs/AlGaAs," Appl. Phys. Lett., vol. 59, pp. 888-890 1991.

[5] L. Buydens, P. Demeester, Z. Yu, and P. Van Daele, "High contrast/low voltage normally-on InGaAs/AlGaAs asymmetric Fabry-Pero modulator," IEEE Photon. Technol. Lett., vol. 3, pp. 1104-1106, 1991.

[6] D. Mahgerefteh, C. M. Yang, L. Chen, K. Hu, W. Chen, E. Garmire, and A. Madhukar, "Picosecond time-resolved measurements of electroabsorption in an InGaAs/GaAs multiple quantum well p-i-n modulator," Appl. Phys. Lett., vol. 61, pp. 2592-2594, 1992.

[7] Li Chen, K. C. Rajkumar, and A. Mahukar, "Optical absorption behavior of strained InxGa1-xAs/GaAs $(100)(x<0.25)$ multiple quantum well structures grown via molecular beam epitaxy," Appl. Phys. Lett., vol. 57 , pp. $2478-2480,1990$

[8] G. Ji, D. Huang, U. K. Reddy, T. S. Henderson, R. Houdre, and H. Morkoc, "Optical investigation of highly strained InGaAs/GaAs multiple quantum wells," $J$. Appl. Phys., vol. 62, pp. 3366-3373, 1987.

[9] J. E. Cunningham, K. W. Goosen, M. Williams, and W. Y. Jan "Pseudomorphic InGaAs/GaAsP quantum well modulators on GaAs," Appl. Phys. Lett., vol. 60, pp. 727-729, 1992.

[10] J. W. Kim, C. W. Chen, T. J. Vogt, L. M. Woods, G. Y. Robinson, and D. L. Lile, "Strained layer InxGa1-xAs/GaAs and InxGa1-xAs/InyGal-yP multiple-quantum-well optical modulators grown by gas-source MBE,' IEEE Photon. Technol. Lett., vol. 5, pp. 987-989, 1993.

[11] T. H. Wood, J. Z. Pastalan, C. A. Burrus, B. C. Johnson, B. J. Miller, J. L. de Miguel, U. Koren, and M. G. Young, "Electric field screening by photogenerated holes in multiple quantum wells: A new mechanism for absorption saturation,"' Appl. Phys. Lett., vol. 57, pp. 1081-1083, 1990

[12] J. A. Cavaillès, D. A. B. Miller, J. E. Cunningham, P. Li Kam Wa, and A. Miller, "Simultaneous measurements of electron and hole sweepout from quantum wells and modeling of photoinduced field screening dynamics,"' IEEE J. Quantum Electron., vol. 28, pp. 2486-2497, 1992.
[13] T. Sizer II, R. E. LaMarche, and T. K. Woodward, "Point source heating effects in multiple quantum well modulators," Appl. Phys. Lett., vol. 61, pp. $420-422,1992$

[14] K. W. Goossen, J. E. Cunningham, M. B. Santos, and W. Y. Jan, "Measurement of modulation saturation intensity in strain-balanced, undefected InGaAs/GaAsP modulators operating at $1.064 \mu \mathrm{m}$," Appl. Phys. Lett., vol. 63, pp. 515-517, 1993.

[15] K. W. Goossen, L. M. F. Chirovsky, R. A. Morgan, J. E. Cunningham, and W. Y. Jan, "High-power extremely shallow quantum-well modulators," IEEE Photon. Technol Lett, vol. 3, pp. 448-450, 1991.

[16] H. Schneider and K. v. Klitzing, "Thermionic emission and gaussian transport of holes in a GaAs/AlGaAs multiple quantum well structure," Phys. Rev. B, vol. 38, pp. 6160-6165, 1988.

[17] G. Van de Walle, "Band line up and deformation potential in the model-solid theory," Phys. Rev. B, vol. 39, pp. 1871-1883, 1989.

[18] H. C. Casey Jr. and M. B. Panish, Heterostructure Lasers. New York: Academic, 1978.

[19] A. Miller, R. J. Manning, P. K. Milsom, D. C. Hutchings, D. W. Crust, and $\mathrm{K}$. Woodbridge, "Transient grating studies of excitonic optical nonlinearities in GaAs/AlGaAs multiple-quantum-well structures," $J$. Opt. Soc. Amer. B, vol. 6, pp. 567-578, 1988.

M. E. Watson was born in Great Bend, KS on October 20, 1969. He received the B.S.E.E. and M.S.E.E. degrees from Colorado State University, Fort Collins, CO, in 1992 and 1995 , respectively.

$\mathrm{He}$ is a member of the Optoelectronics Computing Systems Center. The work in this paper was for partial fulfillment of his Masters degree.

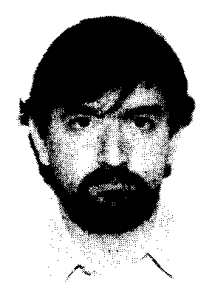

J. L. A. Chilla was born in Lomas de Zamora Argentina in 1960. He received the degrees of Licenciado en Fisica in 1988 and Doctor en Fisica in 1991 from the Universidad de Buenos Aires. He was awarded the 1993 Fundacion Ciencias Exactas y Naturales Prize for the most outstanding doctoral dissertation in the department of Physics.

From 1987 to 1992, he was on a doctoral fellowship with the Comision Nacional de Energia Atomica in Argentina, where he worked on the measurement of ultrashort light pulses in amplitude and phase. In 1992, he joined the Electrical Engineering Department a Colorado State University. His areas of research interest include: lasers, ultrafast phenomena and measurement techniques and the physics behind optoelectronic devices.

J. J. Rocca, photograph and biography not available at the time of publication.

J.-W. Kim, photograph and biography not available at the time of publication.

D. L. Lile, photograph and biography not available at the time of publication.

T. J. Vogt, photograph and biography not available at the time of publication.

G. Y. Robinson (S'66-M'69-SM'93) received the B.E.S. degree at the University of Texas, Austin in 1965 and the M.S. degree in 1967 and the Ph.D. degree in 1969 , both at the University of Califomia, Berkeley.

From 1970 to 1984, he was an Assistant Professor, Associate Professor, and Professor of Electrical Engineering at the University of Minnesota, Minneapolis where he conducted research on electrical and metallurgical characterization of metal-semiconductor contacts on Si and the III-V semiconductors. During 1980, he was on sabbatical leave at Perkin-Elmer Corporation conducting research in molecular beam epitaxy (MBE). In 1984, he started a new research group at Colorado State University, where he is currently a Professor of Electrical Engineering and involved in the growth of III-V heterostructures by gas-source MBE.

Dr. Robinson is a member of the AVS, has taught short courses in the USA and Europe, and has served on organizing committees for numerous conferences on the HI-V semiconductors. 